\title{
Immunocytochemical Studies of the Peptidergic Content of Fibers and Terminals Within the Lateral Spinal and Lateral Cervical Nuclei ${ }^{1}$
}

\author{
GLENN J. GIESLER, JR., ${ }^{2}$ AND ROBERT P. ELDE \\ Department of Anatomy, University of Minnesota, Minneapolis, Minnesota 55455
}

\begin{abstract}
As part of a series of studies in which we are attempting to determine the roles of the lateral spinal (LSn) and lateral cervical (LCn) nuclei in somatic sensation, we have examined the fibers and terminals within these nuclei in the rat using the indirect immunofluorescence technique. Eleven antisera were used. Within the LSn, antisera against dynorphin 1-8 (DYN), substance $P$ (SP), and Met-enkephalin (ENK) produced labeling of a large number of processes in all segmental levels examined. Processes labeled with these antisera frequently apposed the cell bodies and dendrites of LSn neurons. Antisera against somatostatin (SOM) and FMRF$\mathrm{NH}_{2}$ (FMRF) labeled smaller numbers of processes within the LSn. Few, if any, processes in the LSn were labeled using antisera against serotonin, cholecystokinin octapeptide, oxytocin, neurotensin, corticotrophin-releasing factor, and vasoactive intestinal polypeptide. In contrast to the LSn, the LCn contained virtually no labeled processes irrespective of the antiserum employed. An area was found adjacent to the LCn in the medial portion of the dorsal lateral funiculus (DLf) of $\mathrm{C} 2$ that resembled the LSn in several of its anatomical characteristics: (1) like the LSn, the medial portion of the $\mathrm{C}_{2}$ DLf contained small multipolar neurons; (2) it was similar to the LSn in its medial-lateral extent; and (3) following staining with each antiserum, the LSn and the medial DLf of C2 contained a similar number of labeled processes. The peptide-containing area in the medial DLf of $\mathrm{C} 2$ was found to be continuous with the LSn. We therefore propose that this region is a rostral extension of the LSn. The relative abundance of immunoreactive processes in apposition to neurons within the LSn and LCn was confirmed by observers who were unaware of the antiserum employed or the nucleus being evaluated. These findings support our previous suggestion that the DLf in the rat contains two functionally and anatomically distinct nuclei. The LCn of the cat was also examined after immunostaining with DYN, SP, ENK, SOM, and FMRF antisera. A small number of fibers and terminals were labeled by SP antiserum within the medialmost part of the LCn. The remaining antisera failed to label processes in the LCn.
\end{abstract}

Received July 23, 1984; Revised November 26,

Accepted November 28, 1984

\footnotetext{
'We would like lo lhark Ms. Anne Madsen and Mr. Paul Carey for their excellent technical assistance. We also thank Drs. R. Ho and E. Weber for providing antisera. These studies were supported by Grants DA 02148 and NS 17540.

${ }^{2}$ To whom correspondence should be addressed, at Department of Anatomy, 4-135 Jackson Hall, University of Minnesota, Minneapolis, MN 55455.
}

In carnivores, the lateral cervical nucleus $(L C n)$ is a large island of cell bodies located within the dorsal portion of the lateral funiculus (DLf) of spinal segments C1 to C3 (Rexed and Brodal, 1951; Craig and Burton, 1979; cf. also review by Boivie, 1984). The LCn is thought to play a role in nociception since a large percentage of its ascending spinal afferent fibers are differentially responsive to noxious mechanical, thermal, and chemical stimulation (Brown and Franz, 1969; Cervero et al., 1977; Hong et al., 1979). The size of the LCn has been shown to vary dramatically among the more than 45 mammalian species in which it has been examined (Rexed, 1951; Seki, 1962, Kircher and Ha, 1968; Truex et al., 1970). The LCn is largest in carnivores and very small, if present, in primates (Rexed and Brodal, 1951; Kircher and $\mathrm{Ha}, 1968$; Truex et al., 1970; Craig and Burton; 1979). Several early workers (e.g., Rexed, 1951; Seki, 1962) failed to locate an LCn in the rat "in the same form or location" (Rexed, 1951) as that identified in carnivores. However, Gwyn and Waldron (1968, 1969) and Waldron (1969) discovered a number of neurons located within the DLf at all levels of the spinal cord in rats, other rodents, and an insectivore. On the basis of the apparent morphological uniformity of these neurons at all levels of the cord, Gwyri and Waldron $(1968,1969)$ concluded that they Iorm a functionally homogeneous cell column homologous to the LCn. They named this cell column the "nucleus of the dorsal lateral funiculus."

More recent studies have shown, however, that the organization of the cells in the DLf of the rat is more complex than originally envisioned. The cell bodies in the DLf form at least two morphologically and functionally distinct nuclei, one in C2 and the other in C3 and below. We (Giesler et al., 1979a) reported that injections of horseradish peroxidase (HRP) into the ventrobasal thalamus of the rat did not label DLf neurons at all spinal levels uniformly. More than $85 \%$ of the labeled cells within the DLf were confined to segment $\mathrm{C} 2$; the majority of the remaining labeled DLf neurons were within C3 and C1 (Giesler et al., 1979a; Baker and Giesler, 1984). In singleunit studies, all recorded neurons in the DLf of $\mathrm{C} 2$ were found to be responsive to hair movement within large receptive fields that frequently covered half the body surface or more (Giesler et al., 1979b). Twenty-seven percent of these neurons were also shown to respond to noxious cutaneous stimuli (Giesler et al., 1979b). The similarity of the response properties, projections, and morphological characteristics of the neurons in the DLf of segment $C 2$ to those of neurons in the LCn in other species (Rexed and Brodal, 1951; Craig and Tapper, 1978; Craig and Burton, 1979) led us to propose that these neurons constituted the LCn of the rat. In contrast to cells in the DLf of $\mathrm{C}_{2}$, neurons within the DLf below $\mathrm{C} 2$ in the rat can only very rarely be driven by either noxious or innocuous cutaneous stimulation. We could not activate neurons in the DLf of C1 and C3 with any cutaneous stimulus (Giesler et al., 1979b). Menétrey et al. (1980) reported that the overwhelming majority of cells in the DLf of the lumbar enlargement could not be driven by any form of cutaneous stimulation. These workers did report, however, that a minority of such cells were responsive to movement of joints and deep tissue. Also in contrast to DLf cells in C2, DLf cells in cord levels below C2 
have been shown to project only as high as mesencephalic levels (Giesler et al., 1979a, 1981; Menétrey et al., 1980, 1982).

Thus, the demonstrated differences between the neurons of the lateral spinal nucleus (LSn) and LCn indicate that the singular name "nucleus of the dorsal lateral funiculus" should be discarded. In this report the following nomenclature is used: (1) the cells in the lateral part of the DLf of C2 are referred to as the "lateral cervical nucleus" $(\mathrm{LCn}) ;(2)$ since previous and present findings suggest a uniform morphology of neurons within the DLf of segments below C2, we refer to them as the "lateral spinal nucleus" (LSn; cf. Ljungdahl et al., 1978); and (3) and LSn and LCn together are referred to as the nuclei of the dorsal lateral funiculus.

The present report extends an on-going series of studies in which we are attempting to determine the roles of the LCn and LSn in somatic sensation. In the present study, we have immunocytochemically examined and compared the peptidergic content of the fibers and terminals within both the LS̀n and LCn using a variety of neuropeptide antisera. Many of these neuropeptides are thought to be involved in the transmission of somatic sensory information at other levels of the CNS.

\section{Materials and Methods}

Eight Sprague-Dawley rats (200 to $250 \mathrm{gm}$ ) and two adult cats were anesthetized with pentobarbital and perfused intracardially with saline or calcium-free Tyrode's solution followed by a low $\mathrm{pH}(6.5)$ fixative solution containing $4 \%$ paraformaldehyde in $0.1 \mathrm{M}$ Sorenson's phosphate buffer. A second $4 \%$ paraformaldehyde solution at a high $\mathrm{pH}(9.5)$ was then infused for $30 \mathrm{~min}$. A number of spinal cord segments including $\mathrm{C2}, \mathrm{C} 4, \mathrm{C} 7, \mathrm{~T} 8$, and $\mathrm{L} 5$ were identified, removed, and immersed for an additional $90 \mathrm{~min}$ in high $\mathrm{pH}$ fixative. Tissue was stored in $5 \%$ sucrose in $0.1 \mathrm{~m}$ Sorenson's phosphate buffer for 1 to 3 days.

Tissue sections were cut at 10 to $15 \mu \mathrm{m}$ using a cryostat-microtome. Tissue sections were placed on gelatin-coated slides, removed from the cryostat, and warmed to room temperature. Antisera raised against Metenkephalin (ENK) (Elde et al., 1976; Micevych and Elde, 1980), substance $P$ (SP; obtained as a gift from Dr. R. Ho; see Ho and DePalatis, 1980), dynorphin 1-8 (DYN; Peninsula Laboratories, Belmont, CA, and as a gift from E. Weber), serotonin (5-hydroxytryptamine, 5-HT) (Maley and Elde, 1983), somatostatin (SOM) (Arimura et al., 1978; Elde et al., 1978) cholecystokinin octapeptide (CCK-8) (Sasek et al., 1984), oxytocin (OXY) (R. Elde, unpublished data), neurotensin (NT; Immuno Nuclear Corp., Stillwater, MN), FMRF-NH ${ }_{2}{ }^{3}$ (FMRF) (Sasek et al., 1984), corticotrophin-releasing factor (CRF) (Cummings et al., 1983), and vasoactive intestinal polypeptide (VIP; Immuno Nuclear Corp.) werc used in these studies. After rehydration of the tissue with phosphatebuffered saline (PBS), the primary antiserum was diluted in PBS $/ 0.3 \%$ Triton $X-100$ (antiserum diluent) and applied to the tissue. The tissue was incubated for 15 to $20 \mathrm{hr}$ in a hurnid environment at $4^{\circ} \mathrm{C}$. The dilution of the primary antisera ranged from $1 / 100$ to $1 / 400$. After rinsing the primary antiserum from the tissue with PBS, a fluorescein-labeled secondary antiserum (directed against immunoglobulins from the species providing the primary antiserum) in antiserum diluent was applied and the tissue was incubated in a humid environment for $30 \mathrm{~min}$ at $37^{\circ} \mathrm{C}$. After rinsing with PBS, the tissue was counterstained for Nissl substance with ethidium bromide (Schmued et al., 1982), coverslipped with PBS/glycerine, and examined using a fluorescence microscope equipped with reflected illumination.

To acquire an independent evaluation of the relative abundance of peptidecontaining fibers and terminals in apposition to neurons within the nuclei, the five antisera that produced the richest labeling of fibers within the LSn (DYN, SP, ENK, SOM, FMRF) were analyzed using a blind rating procedure. Three individuals who have experience evaluating immunofluorescent material were asked to rate the density of labeled fibers and terminals in apposition to the somata and/or proximal dendrites of counterstained LSn and LCn neurons. A five-point scale was used in which a rating of 0 was assigned when no labeled fibers were seen in apposition to cells within the nuclei and numbers 1 to 4 were given for increasingly richer labeling. The raters were not informed as to the peptide or nucleus being evaluated. Ratings for different peptides and cord regions were compared statistically using the G-test of indepen-

${ }^{3}$ FMRF is a four-amino acid peptide originally isolated in mollusks (Price and Greenberg, 1977). Although FMRF is not found in mammals, antisera raiscd against it cross-react with the mammalian brain peptide, neuropeptide $Y$ (Sasek and Elde, 1985). dence (Sokal and Rohlf, 1981). In all cases, $p<0.05$ was used to indicate a significant difference.

Immunohistochemical specificity controls were prepared by incubating an antiserum with an excess of the homologous antigen prior to incubation of the tissue in the antiserum. In addition, the antisera were screened in a model immunohistochemical system in which neuropeptides were covalently bound with vapor phase formaldehyde to filter paper (Larsson, 1981). In order to be considered specific, an antiserum at its working dilution for brain immunohistochemistry demonstrated intense staining of $10-\mathrm{pmol}$ blots of the homologous peptide and, at the same time, produced no staining above background on 1-nmol blots of all other peptides. According to these criteria, all antisera used in these studies produced specific staining (Sasek et al., 1984).

\section{Results}

Morphological characteristics of LSN and LCn neurons. Very early in these studies it became apparent that the cells of the LSn and LCn differ morphologically. In Figure 1, a number of neurons from LSn and LCn are illustrated in material counterstained with ethidium bromide. In transverse sections, LSn neurons frequently had multipolar or fusiform cell bodies that gave rise to dendrites with no apparent preferred orientation (Fig. 1, $A$ to $C$ ). In contrast, neurons in the LCn had round cell bodies (Fig. 1, $D$ and $E$ ). These neurons have been shown previously to have dendrites that are oriented longitudinally (Giesler et al., 1979a).

Immunocytochemically determined content of fibers and terminals within the LSn. Application of antisera against DYN, SP, or ENK produced very intense staining of processes within the LSn at all examined levels of the cord. Staining with these antisera labeled a large number of small processes that frequently surrounded the cells of the LSn. In many cases, the green fluorescent matcrial surrounding the cell bodies and dendrites was sufficient to allow visualization of many long dendritic branches (Figs. 1, $A$ to $C$, and Fig. 2, DYN L5). The labeled fibers and terminals frequently surrounded many, if not all, LSn neurons visible in a section (Fig. 1, $A$ to $C$ ).

Less intense staining was produced by antisera against SOM or FMRF (Fig. 2). With these antisera, neuronal perikarya and dendrites were most frequently apposed by a small number of labeled processes. Many cells within the LSn appeared to have no labeled fibers apposing them. Antisera against CCK-8, VIP, 5-HT, OXY, and CRF labeled few, if any, processes within the LSn.

Each antiserum used in this study labeled a consistent number of LSn fibers within all examined segments. This feature is depicted in Figure 2 in which labeling in the LSn produced by five antisera is shown in L5 (Fig. 2, left) and C7 (Fig. 2, right). The density of labeling in the LSn at thoracic levels was comparable. The morphological characteristics of the fibers and terminals labeled with an individual antiserum also appeared to be consistent throughout the length of the cord below $\mathrm{C} 2$.

Clear differences could often be seen in the morphological characteristics of the individual processes within the LSn that were stained by the different antisera. The differences in the appearance of the fibers and terminals stained with DYN and SP antisera were perhaps most striking. Processes within the LSn labeled with SP antisera appeared to be very small, often so small that individual labeled elements could not be clearly discerned (Figs. $1 \mathrm{C}$ and 2). SP-labeled processes were seen uniformly across the extent of the nucleus (Fig. 2). The small size and large numbers of these SP. positive fibers made the LSn appear to be enshrouded in a green fog of labeled processes. In contrast, immunostained fibers and terminals in the marginal zone and substantia gelatinosa adjacent to the LSn often appeared as larger, individual stained elements (Figs. $1 C$ and 2). DYN antisera labeled processes within the LSn that were seen as individual, intensely stained elements and were, therefore, larger than SP-stained processes. DYN-positive elements appeared to be less uniformly distributed and fewer in number than SP-labeled processes. However, DYN-immunoreactive fibers were frequently found in apposition to cell bodies and dendrites. Antisera against ENK, SOM, and FMRF also labeled processes that appeared to be very small (Fig. 2). 

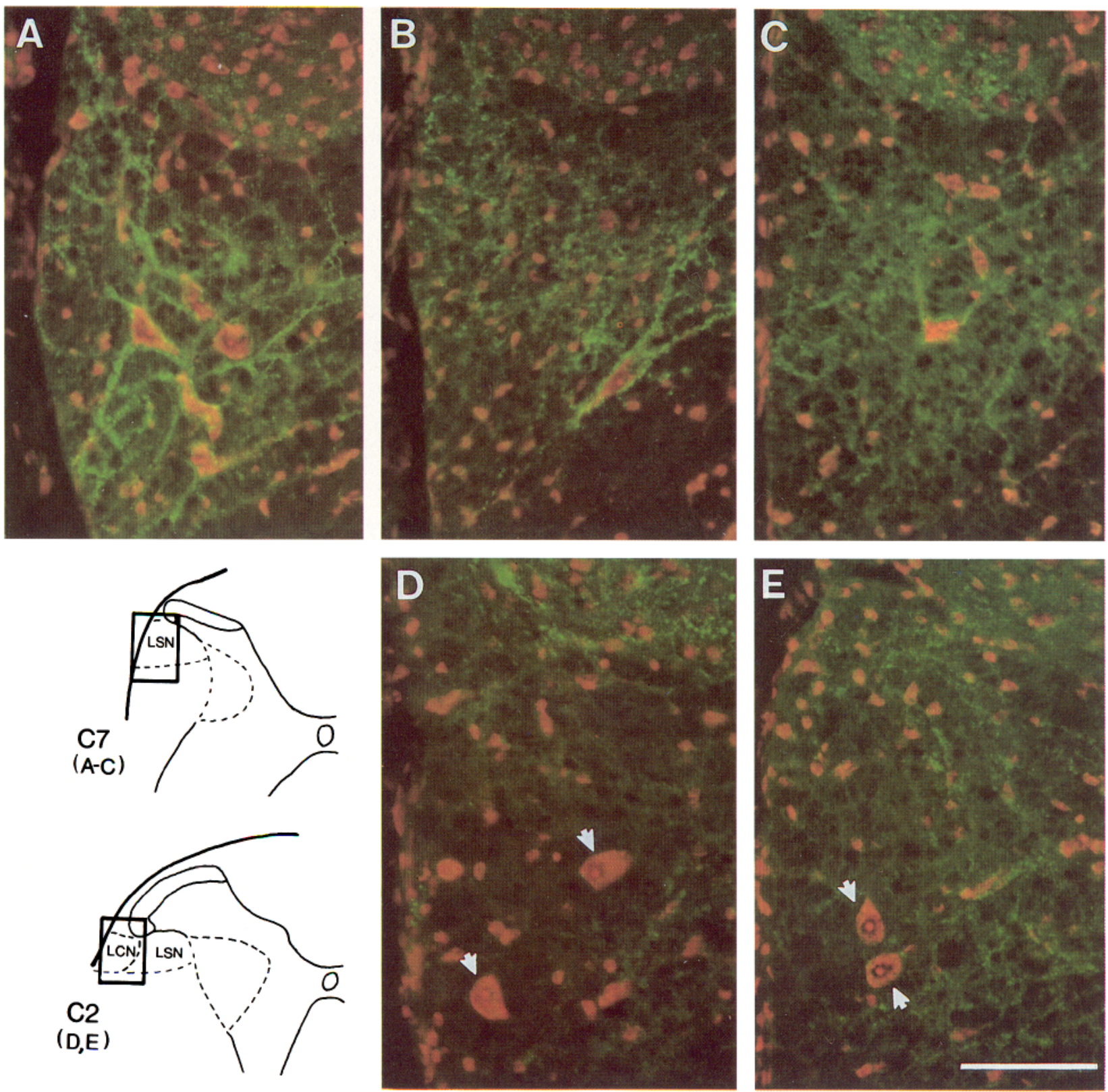

Figure 1. Immunocytochemical demonstration of the peptidergic content of fibers and terminals within the LSn and LCn. In $A$ and $B$, DYN-immunostained fibers are seen apposed to LSn neurons. In these and the remaining panels the dorsal horn is at the top and the lateral edge of the cord is to the left. In $C$, the dendrites of an LSn neuron are apposed by a large number of small SP-immunoreactive processes. The paucity of immunostaining in the LCn is depicted in $D(E N K)$ and $E(S P)$. Note in $E$ the immunoreactive fibers in the C2 LSn medial to the LCn. The areas of the cord illustrated in the photomicrographs are depicted in the line drawings at lower left. Ethidium bromide counterstain. Scale bar $=100 \mu \mathrm{m}$.

Immunocytochemically determined content of processes in the DLf of segment C2. Application of antisera against DYN, SP, and ENK labeled a large number of fibers and terminals in the medial part of the DLf of C2. Unlike the cell bodies found in the lateral part of the DLf at this level, neurons in the medial DLf had small multipolar and fusiform cell bodies. Each antiserum labeled processes in the medial portion of the DLf of C2 that appeared to be similar in quantity and appearance to the processes labeled within the LSn at other cord levels (cf. labeling in Figs. 2 and 3). In nearly horizontal sections of segments C4 to C2 stained for SP and ENK (not illustrated), the LSn was seen in the lateral funiculus of $\mathrm{C} 4$ as a column of multipolar cells encrusted with a number of immunoreactive processes. In C3 and $\mathrm{C} 2$, the LSn was found in a progressively more medial position within the DLf. In caudal C4, the mean and SD of the distance of the lateral border of the LSn from the edge of the cord were $45.2 \pm$ $10.3 \mu \mathrm{m}(N=10)$. In mid-C2, the most lateral immunocytochemically stained fibers were found $120.0 \pm 30.6 \mu \mathrm{m}$ from the edge of the cord $(N=10)$. The difference in these distances is statistically significant (Mann-Whitney $U$ test, $p<0.05$ ). The peptide-containing area in C2 was similar in its medial-lateral extent to the LSn in C4. In C4 the LSn was found to be $196 \pm 23.2 \mu \mathrm{m}(N=10)$ mediolaterally; in $\mathrm{C} 2$, the peptide-containing area was found to be $181 \pm 30.6 \mu \mathrm{m}$ $(N=10)$. These values did not differ significantly. Thus, several features of the nucleus in the medial DLf of C2 suggest that this region is a rostral continuation of the LSn. These include: (1) the cells within the medial DLf of C2 were morphologically similar to LSn neurons, (2) the distribution of immunoreactive fibers in C2 was continuous with the LSn, (3) the medial-lateral extent of the immunoreactive fibers in C2 was similar to that of the LSn, and (4) for each antiserum employed, the relative abundance and morphological characteristics of the immunoreactive fibers in $\mathrm{C} 2$ were similar 

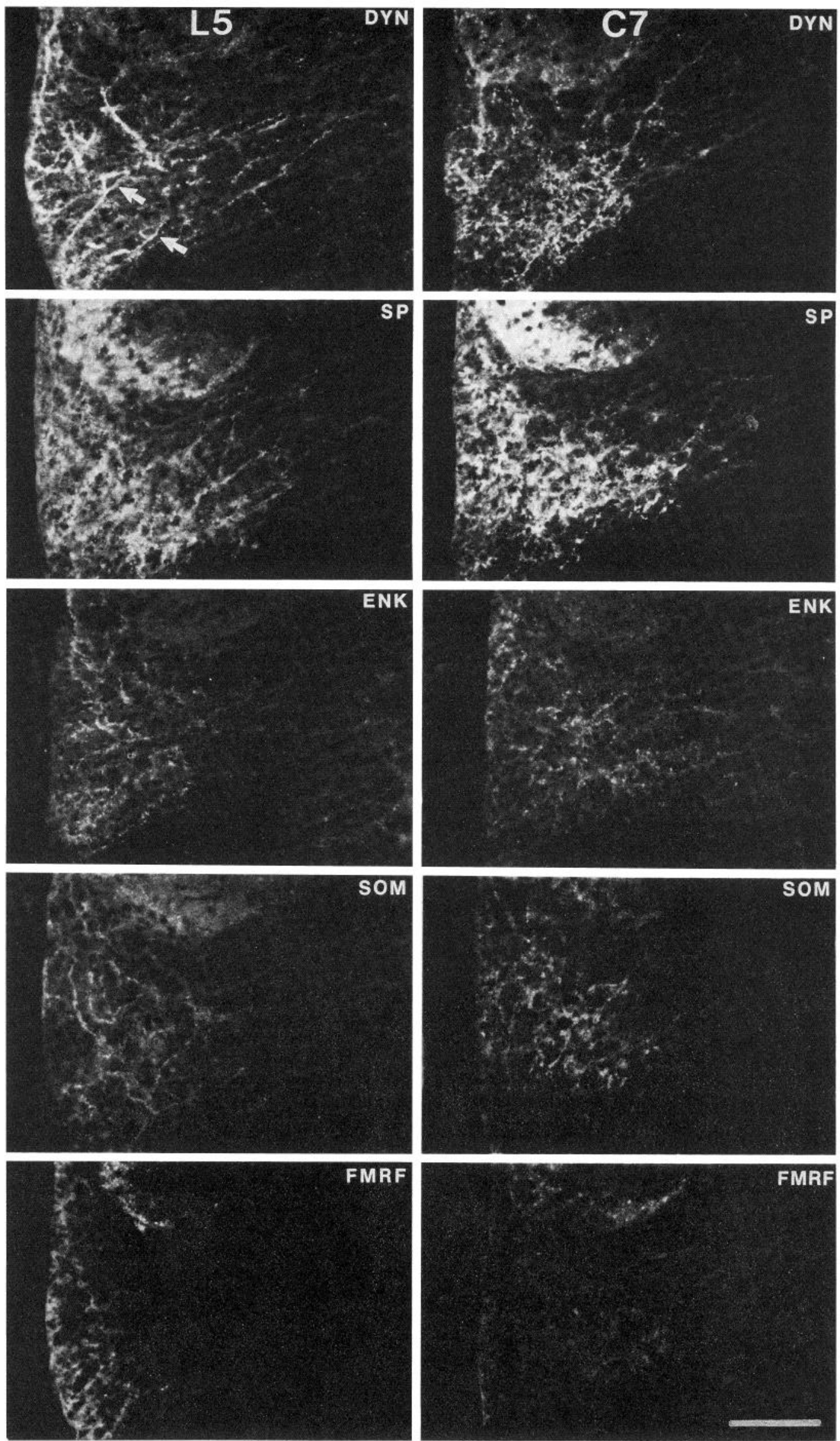

Figure 2. Immunostaining of peptidergic fibers within the LS II. Staining produced by antisera against DYN, SP, ENK, SOM, and FMRF in the LSn of segments L5 (left) and C7 (right) is illustrated. Cells in L5 LSn apposed by DYN-immunoreactive fibers are indicated by arrows. Scale bar $=100 \mu \mathrm{m}$. 

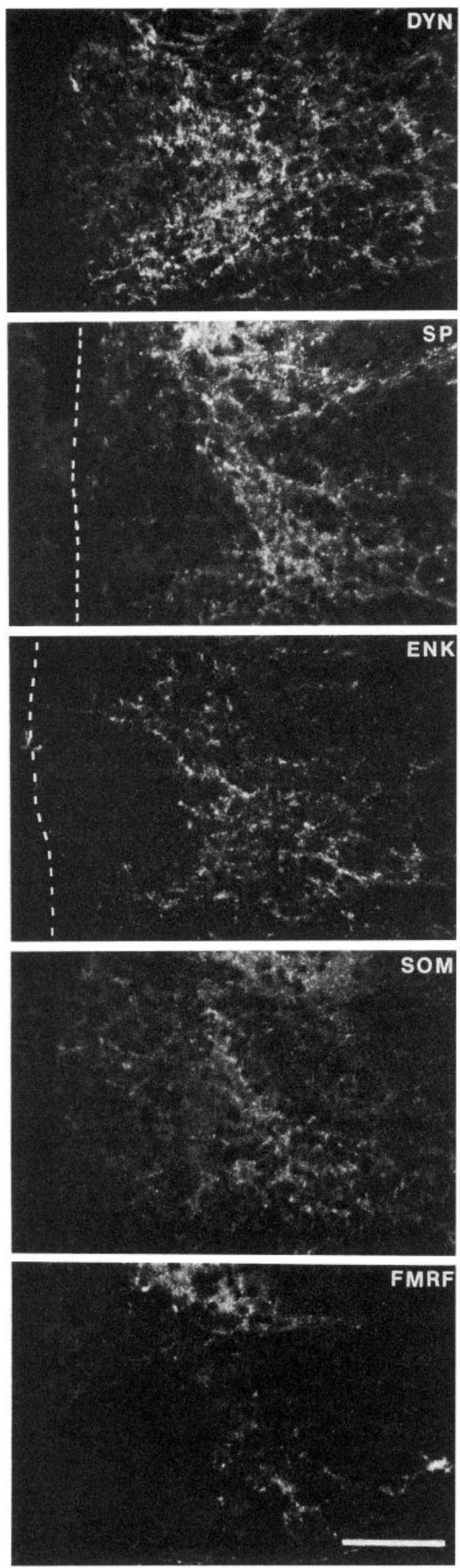

to those of the LSn. We therefore refer to this medial region of the DLf in C2 as the C2 LSn.

None of the antisera employed in this study labeled more than a very small number of fibers in the LCn. As illustrated in Figure 3, this finding was particularly striking in the tissue stained with antisera against DYN, SP, and ENK, for these antisera heavily labeled fibers in the adjacent LSn of C2. In tissue stained with these antisera the LCn can be seen as a "hole" in the labeling within the lateral portion of the C2 DLf.

Ratings of the peptidergic content of the nuclei of the DLf. Ratings of the number of labeled processes within the LSn in L5, C7, and C2 are presented in Figure 4. Sections from each of the three cord levels were stained with antisera against DYN, SP, ENK, SOM, and FMRF and rated. No significant differences were seen in the ratings of processes stained with DYN, SP, SOM, and FMRF across the levels of the LSn. The ratings of the number of ENKlabeled axons in C7 LSn differed significantly from the ratings of the $\mathrm{C} 2 \mathrm{LSn}$. The ratings of neither level, however, differed from those for L5.

As illustrated in Figure 4, the ratings (mean \pm SD) for DYN, SP, ENK, SOM, and FMRF in the LSn in all three cord levels were 3.81 $\pm 0.52,3.47 \pm 0.65,2.94 \pm 1.07,1.75 \pm 1.02$, and $1.58 \pm 0.60$ respectively. In contrast, the ratings of DYN, SP, SOM, and FMRF in the LCn were each $0.17 \pm 0.39$. The ratings for ENK in the LCn were $0.67 \pm 0.49$. The ratings for the LSn differed significantly from those for the LCn for all five peptides examined. The consistent paucity of labeling of processes in the LCn is reflected by the fact that 48 of the 60 evaluated sections through the LCn received a rating of 0 , a rating assigned when not a single labeled process could be seen in apposition to any LCn neurons. In addition, none of the sections of the $L C n$ received a rating higher than 1, regardless of the antiserum used.

Immunocytochemically determined content of processes in the $L C n$ of the cat. As was the case in the rat, application of antisera against DYN, ENK, SOM, or FMRF labeled virtually no fibers within the LCn of the cat (Fig. 5). Antiserum against SP labeled a few fibers within the medialmost part of the cat LCn. No SP labeling was seen in the more lateral portion of LCn. Within the cervical and lumbar enlargements, neither cell bodies nor immunoreactivity (using any of the antisera) were seen in the lateral funiculus, indicating that the LSn in the cat is either considerably smaller than in it is in the rat or is not present. Clear labeling of axons in substantia gelatinosa and the marginal zone $(\mathrm{MZ})$ of the adjacent dorsal horn was produced by antisera against ENK, FMRF, and SP (Fig. 5), indicating that the failure to label processes in LCn of the cat was not the result of faulty immunocytochemical techniques.

Absorption controls. The specificity of the antisera against DYN, $\mathrm{SP}, \mathrm{ENK}, \mathrm{SOM}$, and FMRF was evaluated in absorption control experiments. Application of an excess of the homologous antigen prohibited staining by each antiserum.

\section{Discussion}

Morphologic characteristics of LCn and LSn neurons. In addition to the previously reported differences in response characteristics (Giesler et al., 1979b; Menétrey et al., 1980) and brainstem projections (Giesler et al., 1979a, 1981; Menétrey et al., 1980, 1982) of the neurons that comprise the LSn and LCn, we have found that the morphologic characteristics of the neurons in these nuclei also differ (cf. also Figs. 14 to 17 by G. Grant and B. Robertsson in Boivie, 1984). Neurons in the LCn were most often round in transverse sections. LCn neurons have dendrites that extend primarily longitudinally within the cord (Giesler et al., 1979b). In contrast,

Figure 3. Immunostaining of peptidergic fibers within the LCn and LSn of C2. Staining produced by antisera against DYN, SP, ENK, SOM, and FMRF is illustrated. Note the difference between the LCn and LSn in the density of immunostaining in material stained with DYN, SP, and ENK. Dashed lines indicate the edge of the cord. Scale bar $=100 \mu \mathrm{m}$. 


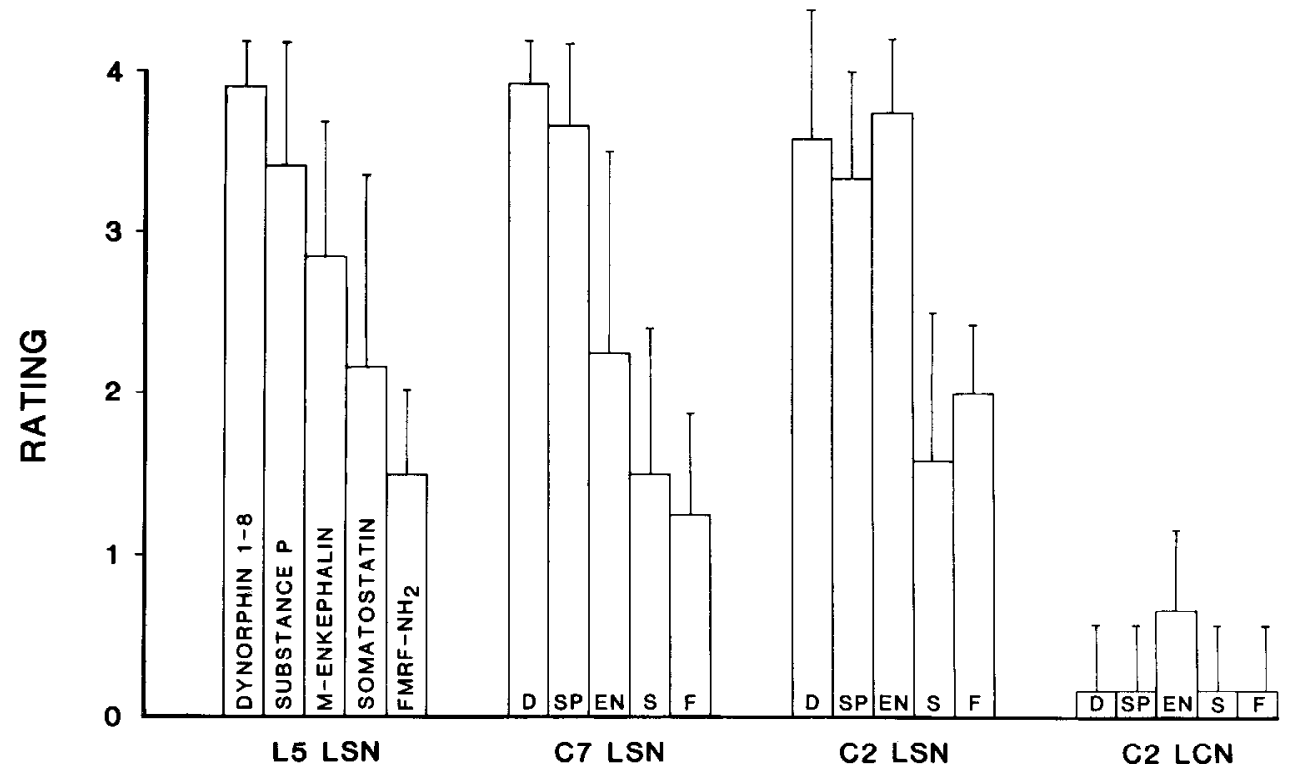

Figure 4. Ratings of immunostained processes in apposition to LSn and LCn neurons. Ratings were made of staining with five antibodies in three levels of the LSn and the LCn.

\section{CORD AREA}

neurons within the LSn had multipolar or fusiform cell bodies that gave rise to dendrites that extended in no apparent preferred direction.

Peptidergic content of the LSn. We have examined the peptidergic content of the nuclei within the DLf in the spinal cord of the rat. Of the 11 antisera employed in the present study, the heaviest labeling of fibers within the LSn was consistently produced by antisera against DYN, SP, and ENK. These antisera frequently produced very heavy labeling of fibers that apposed the cell bodies and dendrites of nearly all LSn neurons. Bresnahan et al. (1984) have recently examined SP- and ENK-immunoreactive fibers and terminals within the LSn at both the light and electron microscopic levels. They reported that a large number of peptide-positive unmyelinated axons and terminals are found within the I Sn. FNK-immunoreactive terminals were found in synaptic contact with dendrites and cell bodies of LSn neurons; SP-stained terminals were found in synaptic contact only with dendrites.

A number of reports have appeared in which immunostaining of processes in the LSn has been noted or illustrated in passing. Our findings confirm a number of these reports. Ljungdahl et al. (1978) first reported that large numbers of fibers in LSn can be richly immunostained for SP. Several reports have confirmed this observation (Barber et al., 1979; Seybold and Elde, 1980; Gibson et al., 1981; Dalsgaard et al., 1982; Senba et al., 1982; Larabi et al., 1983; Bresnahan et al., 1984). Immunostaining with antisera against methionine- or leucine-enkephalin has been shown to produce rich staining in LSn (Simantov et al., 1977; Seybold and Elde, 1980; Gibson et al., 1981; Senba et al., 1982). Previous studies have shown that antisera against SOM or FMRF label a number of LSn fibers (Elde et al., 1978; Seybold and Elde, 1980; Dalsgaard et al., 1981; Senba et al., 1982; Williams and Dockray, 1983; Sasek et al., 1984; Schrøder, 1984). Several studies have shown that very few, if any, fibers are immunostained using antisera against OXY (Seybold and Elde, 1980; Gibson et al., 1981), NT (Seybold and Elde, 1980; Gibson et al., 1981; Senba et al., 1982), CCK-8 (Jancso et al., 1981; Gibson et al., 1981; Dalsgaard et al., 1982; Schrøder, 1983; Sasek ct al., 1984), VIP (Fuji ct al., 1983), and CRF (Schippor ot al., 1983). No evidence was found for the existence of an LSn in the lumbar or cervical enlargements of the cat.

Possible sources of immunostained fibers in LSn. In the first examination of the origins of SP-containing fibers within the LSn, Barber et al., (1979) reported that transection of lumbar dorsal roots markedly reduced the number of labeled fibers in the nucleus ipsilaterally. They also noted that, since combined hemisection of the cord and dorsal rhizotomy failed to eliminate SP labeling in LSn, it was likely that some of the labeled fibers in LSn originated segmentally. In more recent studies, however, the possibility of an input from SP-containing dorsal root fibers to the LSn has been questioned. Larabi et al. (1983) re-examined the effects of dorsal rhizotomy on fibers in the LSn and concluded that, although such operations markedly reduced the number of labeled fibers in the adjacent dorsal horn, little if any reduction was produced in the LSn unless the radicular arteries were obstructed or cut during the rhizotomies. Bresnahan et al. (1984) also showed that rhizotomies have little, if any, effect on SP-immunostained fibers in LSn. In addition, Bresnahan et al. (1984) found that application of HRP to the proximal stumps of cut dorsal roots labeled very few primary afferent terminals in the LSn (the adjacent dorsal horn contained a large number of labeled fibers). The failure to label more than a few fibers in LSn following application of HRP to dorsal roots strongly suggests that the majority of SP-stained fibers within the LSn are not central processes of primary afferent fibers. This finding, of course, also indicates that very few of the fibers within the LSn (whether they contain a peptide or not) originate in dorsal root ganglia. Interestingly, Bresnahan et al. (1984) did find HRP labeling of terminals in LSn when HRP was injected into the dorsal horn, a finding that suggests the existence of a projection from neurons in the adjacent dorsal horn to the LSn. In material available to us from other studies of the dorsal horn in which colchicine had been injected intrathecally to increase the peptidergic labeling of cell bodies (Seybold and Elde, 1980; Sasek et al., 1984), many dorsal horn neurons (see also Ljungdahl et al., 1978; Barber et al., 1979; Bresnahan et al., 1984) and the majority of LSn cell bodies were found to be immunoreactive for SP. It therefore appears that the primary sources of SP-stained fibers in the LSn are the adjacent dorsal horn and, perhaps, the LSn itself. Schrøder (1984) recently demonstrated that SOM-labeled fibers in LSn also originate within the spinal cord. The sources of DYN- and ENK-immunoreactive processes have not been clearly established.

In contrast to the LSn, many arcas of the spinal cord gray matter have been shown to contain dorsal root fibers that are immunoreactive for SP. These areas include the MZ, outer substantia gelatinosa, lateral reticular dorsal horn (lamina $V$ ), and the gray matter around the central canal (Barber et al., 1979; Tessler et al., 1980; Jancso et al., 1981; Larabi et al., 1983). SP is frequently thought to be importantly involved in nociception (Salt and Hill, 1983) and may, in 

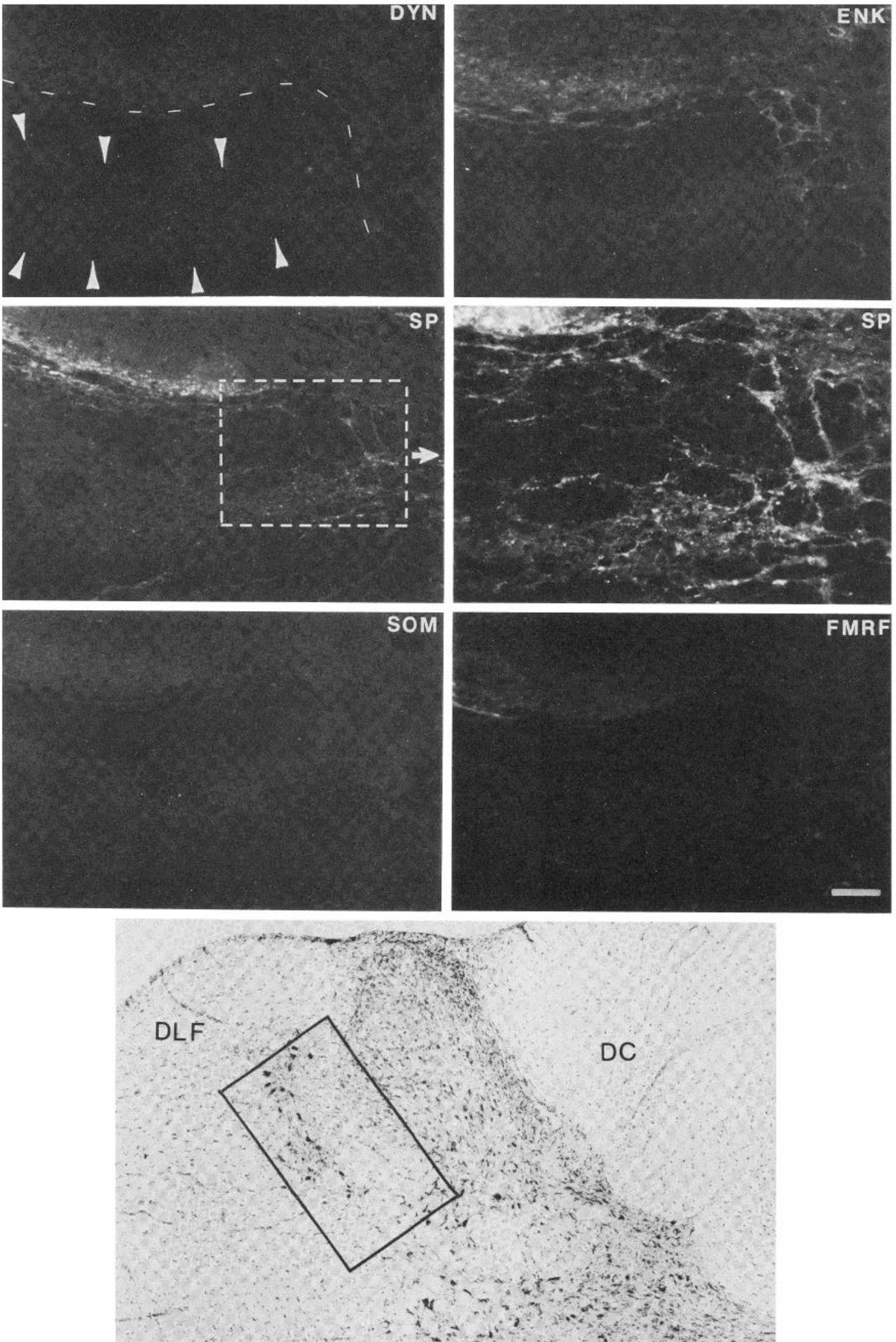

Figure 5. Peptidergic content of fibers in the LCn in C2 of the cat. Antisera against DYN, SOM, and FMRF failed to label any fibers in the LCn. SP and ENK antibodies labeled a small number of fibers in the most medial portions of the LCn. The dashed rectangle over the LCn in the SP-stained material (left) indicates the area illustrated in a higher power micrograph (right). For orientation, a Nissl-stained cross-section of C2 is presented at the bottom; the solid rectangle depicts the area and orientation of the fluorescent micrographs. The dorsal horn is seen at the top in all fluorescent micrographs. Scale bar $=100$ $\mu \mathrm{m}$. 
fact, be contained within nociceptors. Interestingly, all of the regions of the spinal cord gray matter that contain dorsal root fibers which are immunoreactive for SP contain large numbers of neurons that are exclusively or differentially activated by noxious stimuli (Wall, 1967; Christensen and Perl, 1970; Light et al., 1979; Bennett et al., 1980; Nahin et al., 1983). Although the LSn contains a large number of SP-immunoreactive fibers, the neurons within it appear to be responsive to innocuous movement of joints and not to noxious stimuli (Menétrey et al., 1980). Thus, the clear difference between the functional properties of LSn neurons and those of neurons in other areas of the spinal cord that contain SP-immunoreactive fibers may be related to the dittering sources of the SP-containing fibers.

Menétrey et al. (1983) have pointed out that neurons in the $M Z$ and LSn share at least two similar characteristics: both areas contain a large number of neurons that project to the midbrain and both contain fibers that are immunoreactive for some of the same neuropeptides. As a result of these similarities, they made the interesting suggestion that LSn neurons "might represent shifted marginal cells." We feel, however, that several other anatomical as well as physiological findings militate against this idea. These findings include the following. (1) $M Z$ neurons are morphologically distinct from LSn neurons (LSn cells do not resemble any of the major types of $M Z$ neurons including Waldeyer or limitroph cells). (2) Neurons in the $L S n$ are functionally distinct from those in the $M Z$. MZ neurons receive a strong cutaneous input from nociceptors, thermoreceptors, and mechanoreceptors (Christensen and Perl, 1970). Virtually all examined LSn neurons were unresponsive lo any form of culaneous stimulation (Giesler et al., 1979b; Menétrey et al., 1980). (3) Anatomical studies have shown that $M Z$ neurons receive a large, direct input from primary afferent fibers (Snyder, 1982, and references therein), whereas LSn neurons receive little, if any, direct peripheral input (Bresnahan et al., 1984). In support of the findings of Bresnahan et al., (1984), we (K. Cliffer and G. J. Giesler, unpublished observations) have found that lumbar LSn neurons cannot be activated by even intense electrical stimulation of dorsal roots. (4) In at least one respect, the projections of the $M Z$ and $L S n$ clearly differ: $M Z$ neurons in the cervical enlargement frequently project to the thalamus, whereas LSn neurons do not (Giesler et al., 1979a; Kevetter and Willis, 1983). (5) The number of immunoreactive fibers in the LSn and $\mathrm{MZ}$ can also differ. For example, DYN-immunoreactive fibers are far richer in LSn than in $M Z$ (Figs. 1 and 2). (6) As discussed above, the sources and morphology of immunorcactive fibers in the $\mathrm{LSn}$ and $\mathrm{MZ}$ frequently differ.

Immunostaining of fibers and terminals in the DLf of $\mathrm{C} 2$. The organization of cell bodies and immunocytochemically stained fibers within the DLf was found to be more complex in C2 than at other cord levels. The medial half of the DLf in C2 contained a large number of small multipolar cell bodies with dendrites that radiate from their somata with no apparent preferred direction; cell bodies in this area are strikingly similar to those found in the LSn at more caudal levels of the cord. The medial half of the DLf of $C 2$ and the LSn also contain similar densities of immunostained processes. Like the LSn, the medial DLf of C2 contains a large number of SP., DYN-, and ENK-immunostained fibers and terminals in apposition to cell bodies. Again, like the LSn, a moderate to small number of processes in the medial DLf of $\mathrm{C} 2$ was stained with either antisera to SOM or FMRF. Thus, both the morphology of the cell bodies and the immunostaining of the fibers within this area are similar to those found in the LSn at other cord levels. These observations led us to conclude that the neurons within the medial part of the DLf of C2 constitute a rostral continuation of the LSn.

In the cat, no immunoreactive fibers were encountered in the vast majority of the LCn; a small number of fibers were immunostained with antisera against SP in the medialmost portion of the LCn. Craig and his colleagues (Craig and Iapper, 19/8; Craig and Burton, 1979) have shown that the medialmost portion of the LCn of the cat differs from the remainder of the nucleus in several interesting ways. The cells in the more lateral region of the nucleus (more than $90 \%$ of the nucleus) respond exclusively to innocuous cutaneous stimul delivered within relatively small receptive fields (Craig and Tapper, 1978). Over $95 \%$ of the cells within this region project to the contralateral thalamus (Craig and Burton, 1979). In contrast, the medial area of the cat LCn contains a mixture of cells that respond to visceral or noxious cutaneous stimuli within complex receptive fields that frequently cover large areas of the body. Cells in this region are frequently not retrogradely labeled by HRP injections into the thalamus, a finding that has led to speculation that the neurons in this region may be interneurons modulating information ascending to more lateral areas of the LCn (Craig and Burton, 1979). It would be of interest to know the role of $\mathrm{SP}$ in the responses of the neurons that comprise this small but intriguing fraction of the cat $L C$.

A parallel can perhaps be drawn between the present finding that the LCn is virtually devoid of immunostaining for peptides and previous reports demonstrating that many other areas that receive a prominent ascending spinal projection also contain few, it any, peptidergic fibers. Areas such as the bulbar reticular formation, deep layers of the superior colliculus, central lateral nucleus, and the ventrobasal thalamus have all been shown to contain small numbers of peptide-immunoreactive fibers (Elde et al., 1976; Ljundahl et al., 1978; Haber and Elde, 1982). The dorsal horn neurons of origin of the ascending pathways that terminate within these areas are frequently located in regions that contain a large number of peptidergic fibers and terminals (Elde et al., 1978; Ljungdahl et al., 1978; Gibson et al., 1981; Jancso et al., 1981; Ruda, 1982). Thus, the present and previous irmmunocylochernical studies suggest that if neuropeptides affect synaptic transmission within several ascending somatic sensory systems, they are more likely to do so at the level of the spinal cord dorsal horn than in the recipient brainstem nuclei.

\section{References}

Arimura, A., G. Lundqvist, J. Rothman, R. Chang, R. Fernandez-Durango, R. Elde, D. H. Coy, C. Meyers, and A. V. Schally (1978) Radioimmunoassay of somatostatin. Metabolism 1: 1139-1144.

Baker, M. L., and G. J. Giesler, Jr. (1984) Anatomical studies of the spinocervical tract of the rat. Somatosensory Res. 2: 1-18.

Barber, R. P., J. E. Vaughn, J. R. Slemmon, P. M. Salvaterra, E. Roberts, and S. E. Leeman (1979) The origin, distribution, and synaptic relationships of substance $P$ axons in rat spinal cord. J. Comp. Neurol. 184: 331-352.

Bennett, G. J., M. Abdelmoumene, H. Hayashi, and R. Dubner (1980) Physiology and morphology of substantia gelatinosa neurons intracellularly stained with horseradish peroxidase. J. Comp. Neurol. 194: 809-827.

Boivie, J. (1984) Anatomic and physiologic features of the spino-cervicothalamic pathway. In Somatosensory Intergration in the Thalamus, G. Macchi, A. Rustiont, and R. Spreatico, eds., pp. 63-106, Elsevier-North Holland Publishing Co., Amsterdam.

Bresnahan, J. C., R. H. Ho, and M. S. Beattie (1984) A comparison of the ultrastructure of substance-P and enkephalin immunoreactive elements in the nucleus of the dorsal lateral funiculus and laminae I and II of the rat spinal cord. J. Comp. Neurol. 229: 497-511.

Brown, A. G., and D. N. Franz (1969) Responses of spinocervical tract neurons to natural stimulation of identified cutaneous receptors. Brain Res. 7: $231-249$.

Cervero, F., A. Iggo, and V. Molony (1977) Response of spinocervical tract neurones to noxious stimulation of the skin. J. Physiol. (Lond.) 267: 537558

Christensen, B., and E. Perl (1970) Spinal neurons specifically excited by noxious or thermal stimuli: Marginal zone of the dorsal horn. J. Neurophysiol. 33: 293-307.

Craig, A. D., Jr., and H. Burton (1979) The lateral cervical nucleus in the cat: Anatomic organization of cervicothalamic neurons. J. Comp. Neurol. 185: 329-346.

Craig, A. D., Jr., and D. Tapper (1978) Lateral cervical nucleus in the cat: Functional organization and characteristics. J. Neurophysiol. 41: 15111534.

Cummings, S., R. Elde, J. Ells, and A. Lindall (1983) Corticotropin-releasing factor immunoreactivity is widely distributed within the central nervous system of the rat: An immunohistochemical study. J Neurosci. 3: 13551368 .

Dalsgaard, C. J., T. Hökfolt, O. Johansson, and R. Elde. (1981) Somatostatin immunoreactive cell bodies in the dorsal horn and the parasympathetic 
intermediolateral nucleus of the rat spinal cord. Neurosci. Lett. 27: 335339.

Dalsgaard, C. -J., S. R. Vincent, T. Hökfelt, J. M. Lundberg, A. Dahlstrom, M. Schultzberg, G. J. Dockray, and A. C. Cuello (1982) Coexistence of cholecystokinin- and substance P-like peptides in neurons of the dorsal root ganglia of the rat. Neurosci. Lett. 33: 159-163.

Elde, R., T. Hökfelt, O. Johansson, and L. Terenius (1976) Immunohistochemical studies using antibodies to leucine-enkephalin: Initial observations on the nervous system of the rat. Neuroscience 1: 349-351.

Elde, R., T. Hökfelt, O. Johansson, M. Schultzburg, S. Efendic, and R. Luft (1978) Cellular localization of somatostatin. Metabolism 27 (Suppl. 1):1151-1159

Fuji, K., E. Senba, Y. Ueda, and M. Tohyama (1983) Vasoactive intestinal polypeptide (VIP)-containing neurons in the spinal cord of the rat and their projections. Neurosci. Lett. 37: 51-55.

Gibson, S. J., J. M. Polak, S. R. Bloom, and P. D. Wall (1981) The distribution of nine peptides in rat spinal cord with special emphasis on the substantia gelatinosa and on the area around the central canal (lamina $X$ ). J. Comp. Neurol. 201: 65-79.

Giesler, G. J., Jr., D. Menétrey, and A. I. Basbaum (1979a) Differential origins of spinothalamic tract projections to medial and lateral thalamus in the rat. J. Comp. Neurol. 184: 107-126.

Giesler, G. J., Jr., G. Urca, J. T. Cannon, and J. C. Liebeskind (1979b) Response properties of neurons of the lateral cervical nucleus in the rat. J. Comp. Neurol. 186: 65-78.

Giesler, G. J., Jr., H. R. Spiel, and W. D. Willis (1981) Organization of spinothalamic tract axons within the rat spinal cord. J. Comp. Neurol. 195: 243-252

Gwyn, D. G., and H. A. Waldron (1968) A nucleus in the dorsolateral funiculus of the spinal cord of the rat. Brain Res. 10: 342-351.

Gwyn, D. G., and H. A. Waldron (1969) Observations on the morphology of a nucleus in the dorsolateral funiculus of the spinal cord of the guinea-pig, rabbit, ferret and cat. J. Comp. Neurol. 136: 233-236

Haber, S., and R. Elde (1982) The distribution of enkephalin immunoreactive fibers and terminals in the monkey central nervous system: An immunohistochemical study. Neuroscience 7: 1049-1095.

Ho, R., and L. DePalatis (1980) Substance P immunoreactivity in the median eminence of the North American oppossum and domestic fowl. Brain Res. 189: 565-569.

Hong, S. K., K. D. Kniffki, S. Mense, R. F. Schmidt, and M. Wendisch (1979) Descending influences on the responses of spinocervical tract neurones to chemical stimulation of fine muscle afferents. J. Physiol. (Lond.) 290: 129-140.

Jancso, G., T. Hökfelt, J. M. Lundberg, E. Kirally, N. Halasz, G. Nilsson, L. Terenius, J. Rehfeld, H. Steinbush, A. Verhofstad, R. Elde, S. Said, and M. Brown (1981) Immunohistochemical studies on the effect of capsaicin on spinal and medullary peptide and monoamine neurons using antisera to substance $P$, gastrin/CCK, somatostatin, VIP, emkephalin, neurotensin and 5-hydroxytryptamine. J. Neurocytol. 10: 963-980.

Kevetter, G. A., and W. D. Willis (1983) Collaterals of spinothalamic cells in the rat. J. Comp. Neurol. 215: 453-464.

Kircher, C., and $\mathrm{H}$. Ha (1968) The nucleus cervicalis lateralis in primates including the human. Anat. Rec. 160: 376.

Larabi, Y., B. Berger, J. M. Besson, M. C. Lombard, and M. Gay (1983) Substance $P$ in the dorsal horns of partially deafferented rats. In Advances in Pain Research and Therapy, J. J. Bonica, U. Lindblom, and A. Iggo, eds., Vol. 5, pp. 277-282, Raven Press, New York.

Larsson, L. 1. (1981) A novel immunocytochemical model syslem for specificity and sensitivity screening of antisera against multiple antigens. J. Histochem. Cytochem. 29: 408-410.

Light, A. R., D. L. Irevino, and E. R. Perl (1979) Morphological teatures of functionally defined neurons in the marginal zone and substantia gelatinosa of the spinal dorșal horn. J. Comp. Neurol. 186: 151-171.

Ljungdahl, A., T. Hökfelt, and G. Nilsson (1978) Distribution of substance Plike immunoreactivity in the central nervous system of the rat. I. Cell bodies and nerve terminals. Neuroscience 3: 861-943.

Maley, B., and R. Elde. (1983) Immunocytochemical localization of putative neurotransmitters within the feline nucleus tractus solitarius. Neuroscience 7: 2469-2490.

Menétrey, D., A. Chaouch, and J. M. Besson (1980) Location and properties of dorsal horn neurons at origin of spinoreticular tract in lumbar enlargement of the rat. J. Neurophysiol. 44 : 862-877.
Menétrey, D., A. Chaouch, D. Binder, and J. M. Besson (1982) The origin of the spinomescencephalic tract in the rat: An anatomical study using the retrograde transport of horseradish peroxidase. J. Comp. Neurol. 206: 193-207.

Menétrey, D., F. Roudier, and J. M. Besson (1983) Spinal neurons reaching the lateral reticular nucleus as studied in the rat by retrograde transport of horseradish peroxidase. J. Comp. Neurol. 220: 439-452.

Micevych, P., and R. Elde (1980) Relationship between enkephalinergic neurons and the vasopressin-oxytocin neuroendocrine system of the cat: An immunohistochemical study. J. Comp. Neurol. 190: 135-146.

Nahin, R. L., A. M. Madsen, and G. J. Giesler, Jr. (1983) Anatomical and physiological studies of the gray matter surrounding the spinal cord central canal. J. Comp. Neurol. 220: 321-335.

Price, D. A., and M. J. Greenberg (1977) Structure of a molluscan cardioexcitatory neuropeptide. Science 197: 670-671.

Rexed, B. (1951). The nucleus cervicalis lateralis, a spinocerebellar relay nucleus. Acta Physiol. Scand. (Suppl.) 89: 67-68.

Rexed, B., and A. Brodal (1951) The nucleus cervicalis lateralis, a spinocerebellar relay nucleus. J. Neurophysiol. 14: 399-407.

Ruda, M. A. (1982) Opiates and pain pathways: Demonstration of enkephalin synapses on dorsal horn projestion neurons. Science 215: 1523-1525.

Salt, T. E., and R. G. Hill (1983) Neurotransmitter candidates of somatosensory primary afferent fibers. Neuroscience 10: 1083-1103.

Sasek, C. $\wedge$., and R. P. Elde (1985) Distribution of neuropeptide Y like immunoreactivity and its relationship to FMRF-amide-ike immunoreactivity in the sixth lumbar and first sacral spinal cord segments of the rat. $\mathrm{J}$. Neurosci. 5: 1729-1739.

Sasek, C. A., V. S. Seybold, and R. P. Elde (1984) The immunohistochemical localization of nine peptides in the sacral parasympathetic nucleus and the dorsal gray commissure in rat spinal cord. Neuroscience 12: 855-8/3.

Schipper, J., H. W. M. Steinbusch, I. Vermes, and F. J. H. Tilders (1983) Mapping of CRF-immunoreactive nerve fibers in the medulla oblongata and spinal cord of the rat. Brain Res. 267: 145-150.

Schmued, L. C., L. W. Swanson, and P. E. Sawchenko (1982) Some fluorescent counterstains for neuroanatomical studies. J. Histochem. Cytochem. 30: 123-128.

Schrøder, H. A. (1983) Localization of cholecystokinin-like immunoreactivity in the rat spinal cord, with particular reference to the autonomic innervation of pelvic organs. J. Comp. Neurol. 217: 176-186.

Schrøder, H. A. (1984) Somatostatin in the caudal spinal cord: An immunohistochemical study of the spinal centers involved in the innervation of pelvic organs. J. Comp. Neurol. 223: 400-414.

Seki, Y. (1962) Some aspects of comparative anatomy of the spinal cord. Recent Adv. Res. Nerv. Syst. 6: 908-924.

Senba, E., S. Shiosaka, Y. Hara, S. Inagaki, M. Sakanaka, K. Takatsuki, Y. Kawai, and M. Tohyama (1982) Ontogeny of the peptidergic system in the rat spinal cord: Immunohistochemical analysis. J. Comp. Neurol. 208: 5466.

Seybold, V., and R. P. Elde (1980) Immunohistochemical studies of the peptidergic neurons in the dorsal horn of the spinal cord. J. Histochem. Cytochem. 28: 367-370.

Simantov, R., M. J. Kuhar, G. R. Uhl, and S. H. Snyder (1977) Opioid peptide enkephalin: Immunohistochemical mapping in rat centrol nervous system. Proc. Natl. Acad. Sci. U. S. A. 74: 2167-2171.

Snyder, R. L. (1982) Light and electron microscopic autoradiographic study of the dorsal root projections to the cat dorsal horn. Neuroscience 7 : $1417-1437$.

Sokal, R. R., and F. J. Rohlf (1981) Biometry: The Principles and Practice of Statistics in Biological Research, W. H. Freeman and Co., San Francisco.

Tessler, A., E. Glazer, R. Artymyshyn, M. Murray, and M. Goldberger (1980) Recovery of substance $P$ in the cat spinal cord after unilateral lumbosacral deafferentation. Brain Res. 191: 459-470.

Truex, R. C., M. J. Taylor, M. Q. Smythe, and P. L. Gildenberg (1970) The lateral cervical nucleus of cat, dog, and man. J. Comp. Neurol. 139, 93140.

Waldron,. H. A. (1969) The morphology of the lateral cervical nucleus in the hedgehog. Brain Res. 16: 301-306.

Wall, P. (1967) The laminar organization of dorsal horn cells and effects of descending impulses. J. Physiol. (Lond.) 188: 403-423.

Williams, R. G., and G. J. Dockray (1983) Immunohistochemical studies of FMRF-amide-like immunoreactivity in rat brain. Brain Res. 276: 213-229. 